2015

\title{
Properly Colored Notions of Connectivity - A Dynamic Survey
}

Xueliang Li

Nankai University, lxl@nankai.edu.cn

Colton Magnant

Georgia Southern University, cmagnant@georgiasouthern.edu

Follow this and additional works at: https://digitalcommons.georgiasouthern.edu/tag

Part of the Discrete Mathematics and Combinatorics Commons

\section{Recommended Citation}

Li, Xueliang and Magnant, Colton (2015) "Properly Colored Notions of Connectivity - A Dynamic Survey," Theory and Applications of Graphs: Vol. 0 : Iss. 1 , Article 2.

DOI: $10.20429 /$ tag.2015.000102

Available at: https://digitalcommons.georgiasouthern.edu/tag/vol0/iss1/2 


\section{Properly Colored Notions of Connectivity - A Dynamic Survey}

\section{Cover Page Footnote}

Supported by NSFC No. 11371205 and 11531011 


\begin{abstract}
A path in an edge-colored graph is properly colored if no two consecutive edges receive the same color. In this survey, we gather results concerning notions of graph connectivity involving properly colored paths.
\end{abstract}

\title{
Revision History
}

- Revision 1: December, 2016

- Original: December, 2015

If you have corrections, updates or new results which fit the scope of this work, please contact Colton Magnant at coltonmagnant@yahoo.com.

\section{Contents}

1 Introduction $\quad 2$

2 General Results $\quad 3$

3 Connectivity $\quad 5$

4 Complements $\quad 7$

5 Degree Conditions $\quad 7$

5.1 Minimum Degree . . . . . . . . . . . . . . . . . 7

5.2 Degree Sum . . . . . . . . . . . . . . . . . . . . . 9

5.3 Edge Density . . . . . . . . . . . . . . . . . . . . . . . . . . 9

6 Domination $\quad 9$

7 Combinations of Graphs $\quad 10$

8 Operations on Graphs $\quad 10$

9 Random Graphs 12

10 Proper Distance 12

11 Strong Proper Connection 12

11.1 General Results . . . . . . . . . . . . . . . . . . . . . . . . 13

11.2 Operations on Graphs . . . . . . . . . . . . . . . . . . . . . . . . . . . . 14

12 Proper Vertex Connection 14

13 Total Proper Connection 16

14 More Paths $\quad 17$

15 Directed Graphs $\quad 18$

16 Generalizations $\quad 19$

$16.1(k, \ell)$-Rainbow Connectivity . . . . . . . . . . . . . . . 19

16.2 Proper Hamiltonian-Connection . . . . . . . . . . . . . . . . . . . . . . 21

16.3 Proper-Walk Connection . . . . . . . . . . . . . . . . . 22 
16.4 Proper Index . . . . . . . . . . . . . . . . . . . . . . . . . . . 22

16.5 Minimum Spanning Subgraphs . . . . . . . . . . . . . . . 25

\section{Introduction}

An edge-colored graph is said to be properly colored if no two adjacent edges share a color. An edge-colored connected graph $G$ is called properly connected if between every pair of distinct vertices, there exists a path that is properly colored. The proper connection number of a connected graph $G$, defined in [7] and also studied in [1] and [35], is the minimum number of colors needed to color the edges of $G$ to make it properly connected.

When building a communication network between wireless signal towers, one fundamental requirement is that the network is connected. If there cannot be a direct connection between two towers $A$ and $B$, say for example if there is a mountain in between, there must be a route through other towers to get from $A$ to $B$. As a wireless transmission passes through a signal tower, to avoid interference, it would help if the incoming signal and the outgoing signal do not share the same frequency. Suppose we assign a vertex to each signal tower, an edge between two vertices if the corresponding signal towers are directly connected by a signal and assign a color to each edge based on the assigned frequency used for the communication. Then the number of frequencies needed to assign the connections between towers so that there is always a path avoiding interference between each pair of towers is precisely the proper connection number of the corresponding graph.

Aside from the above application, properly colored paths and cycles appear in a variety of other fields including genetics [20,21, 22] and social sciences [17]. There is also a good survey [3] dealing with the case where two colors are used on the edges. More recently, there has also been another survey of the area in Chapter 16 of [4].

Unless otherwise stated, we focus on coloring edges so "coloring" will mean edge-coloring. In most cases, $k$ will generally be used to denote the number of colors used on the edges and $n$ will generally be used to denote the order of $G$, that is, the number of vertices. Also define the color degree $d^{c}(v)$ to be the number of colors on edges incident to $v$. The connectivity of a graph $G$, denoted by $\kappa(G)$, is the minimum order of a set of vertices such that its removal results in at least two components. The chromatic number $\chi(G)$ (and similarly edge chromatic number $\left.\chi^{\prime}(G)\right)$ is the minimum number of colors needed to color the vertices (respectively edges) so that no two adjacent vertices (respectively edges) receive the same color. For all other standard terminology, we refer the reader to [14].

The notion of proper edge-colorings has been extremely popular since the classical work of Vizing [46]. More recently, several works have considered properly colored subgraphs as opposed to looking at the entire graph. See [3] for a survey of work concerning properly colored cycles and paths in graphs and multigraphs.

The definition and study of the proper connection number was inspired by the rainbow connection number, defined by Chartrand et al. in [13]. A path is called rainbow if no two edges in the path share a color. The rainbow connection number of a graph $G$, denoted by $r c(G)$, is the minimum number of colors needed to color the graph so that between each pair of vertices, there is a rainbow path. By replacing "rainbow" with "proper", it is easy to see where the definition of the proper connection number originated. Furthermore, the strong 
rainbow connection number, denoted by $\operatorname{src}(G)$, is the minimum number of colors needed to color the graph so that between every pair of vertices, there is a rainbow colored geodesic (shortest path).

More generally, a graph $G$ is said to be $k$-properly connected if between every pair of distinct vertices, there exist $k$ internally disjoint properly colored paths. The $k$-proper connection number of a $k$-connected graph $G$, denoted by $p c_{k}(G)$, is the minimum number of colors needed to color the edges of $G$ to make it $k$-properly connected. For ease of notation and since many results concern only the 1-proper connection number, we let $p c(G)=p c_{1}(G)$.

\section{General Results}

Clearly $p c(G)=1$ if and only if $G=K_{n}$ and $p c(G)=n-1$ if and only if $G=K_{1, n-1}$. It is also clear that $p c_{k}(G)$ is monotone non-increasing under the operation of edge addition. The graphs $G$ with $p c(G)=n-2$ were classified by Huang et al. Here we let $C_{n}$ and $S_{n}$ denote the cycle and star on $n$ vertices respectively.

Theorem 2.1 ([32]) Let $G$ be a connected graph on $n$ vertices. Then $p c(G)=n-2$ if and only if $G$ is one of the following graphs:

- the unique tree with one vertex of degree $n-2$,

- $C_{3}$,

- $C_{4}$,

- $C_{4}+e$,

- $S_{4}+e$, or

- $S_{5}+e$.

There is an easy lower bound on $p c(G)$ using the maximum number of bridges (cut edges) incident to a single vertex.

Theorem 2.2 ([1]) Let $G$ be a nontrivial connected graph that contains bridges. If $b$ is the maximum number of bridges incident with a single vertex in $G$, then $p c(G) \geq b$.

The bridges also play a role in an upper bound. A block of a graph $G$ is a vertexmaximal 2-edge-connected subgraph. Let $G^{*}$ be the graph constructed from a given graph $G$ be contracting all of the blocks of $G$ to a single vertex each.

Theorem 2.3 ([31]) If $G$ is a connected graph, then $p c(G) \leq \max \left\{3, \Delta\left(G^{*}\right)\right\}$.

Corollary 2.4 ([31]) If $G$ is a connected graph with $n$ vertices and minimum degree $\delta \geq 2$, then $\operatorname{pc}(G) \leq \max \left\{3, \frac{n-1}{\delta+1}\right\}$. Moreover, if $\frac{n-1}{\delta+1}>3$ and $n \geq \delta(\delta+1)+1$, we have that $p c(G)=\frac{n-1}{\delta+1}+1$ if and only if $G \cong S_{r}^{t}$, where $t-1=\delta$ and $r t+1=n$ 
Trees also provide helpful bounds as seen in the following sequence of results.

Proposition 2.1 ([1]) If $T$ is a nontrivial tree, then $p c(T)=\chi^{\prime}(T)=\Delta(T)$.

Proposition 2.2 ([1]) For a nontrivial connected graph $G, p c(G) \leq \min \{\Delta(T)$ : where $T$ is a spanning tree of $G$ \}.

Corollary 2.5 ([1]) If $G$ contains a spanning path, then $p c(G)=2$.

Similarly, unicyclic graphs also have proper connection number almost the maximum degree.

Theorem 2.6 ([36]) If $G$ is a unicyclic graph, then $\Delta(G)-2 \leq p c(G) \leq \Delta(G)$.

In comparing the proper connection number to the rainbow connection number or the chromatic index, the following fact is clear from the definitions.

Fact 2.1 For any connected graph $G, p c(G) \leq \chi^{\prime}(G)$ and $p c(G) \leq \operatorname{rc}(G)$.

On the other hand, the following results show that there can be an arbitrarily large difference between these values.

Proposition 2.3 ([1]) For any pair of positive integers $a, b$ with $2 \leq a \leq b$, there is $a$ connected graph $G$ with $p c(G)=a$ and $\operatorname{rc}(G)=b$.

Proposition 2.4 ([1]) For any pair of positive integers $a, b$ with $2 \leq a \leq b$, there is $a$ connected graph $G$ with $p c(G)=a$ and $\chi^{\prime}(G)=b$.

Regarding complete multipartite graphs, the following result shows that the proper connection number is almost always 2 .

Theorem 2.7 ([1]) If $G$ is a complete multipartite graph that is not a complete graph or a tree, then $p c(G)=2$.

The following results classify the graphs with proper connection number very large, that is, close to the number of edges in the graph. Here $S_{i, j}$ is the double star, that is the tree with exactly two internal vertices of degrees $i$ and $j$ respectively.

Theorem 2.8 ([35]) Let $G$ be a connected graph of size $m \geq 3$. Then $p c(G)=m-1$ if and only if $G$ is the double star $S_{2, m-1}$.

Theorem 2.9 ([35]) Let $G$ be a connected graph of size $m \geq 4$. Then $p c(G)=m-2$ if and only if $G$ is the tree with max degree $m-2$ or $G \in\left\{C_{4}, K_{1,3}+e, K_{1,4}+e\right\}$.

Theorem 2.10 ([35]) Let $G$ be a connected graph of size $m \geq 5$. Then $p c(G)=m-3$ if and only if $G$ is one of the following graphs:

- a tree with $\Delta(G)=m-3$, 
- $G=K_{1, m-1}+$ e where $m \geq 6$,

- a star on $m-3$ edges with the addition of a triangle sharing a vertex with one leaf of the star, or

- one of six specific graphs on at most 6 edges.

Concerning vertex deletion, there is the following result.

Theorem 2.11 ([2]) Let $G$ be a connected graph of order at least 3 . If $v$ is a non-cut-vertex of $G$, then

$$
p c(G)-1 \leq p c(G-v) \leq p c(G)+\operatorname{deg}(v) .
$$

Furthermore both bounds are best possible.

Concerning edge deletion, there is the following result.

Theorem $2.12([2])$ Let $G$ be a connected graph of order at least 3 . If $e$ is an edge of $G$ that is not a bridge, then

$$
p c(G) \leq p c(G-e) \leq p c(G)+2 .
$$

Furthermore both bounds are best possible.

\section{Connectivity}

In [7], several results were proven concerning the proper connection number using connectivity to provide density.

Theorem 3.1 ([7]) If $\kappa(G) \geq 3$, then $p c(G)=2$. If $\kappa(G) \geq 2$, then $p c(G) \leq 3$.

This result was actually proven in a stronger form, to include the following "strong property", providing a special type of proper circuit containing any pair of vertices.

Definition 3.1 (Strong Property) A proper connected graph is said to have the strong property if for every pair of vertices $u$ and $v$, there exist two different (not necessarily disjoint) proper paths, say $P$ and $Q$, from $u$ to $v$ such that the first colors used on each path are different and the last colors used on each path are different.

The sharpness of Theorem 3.1 comes from the graph in Figure 1.

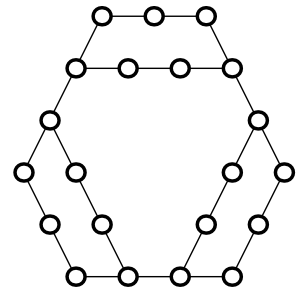

Figure 1: A 2-connected graph with $p c(G)=3$

Theorem 3.1 was recently reproven in [30] using induction on the number of blocks in a connected bridgeless graph. More specifically, the following results were shown. 
Theorem 3.2 ([30]) If $G$ is a connected bridgeless graph, then $p c(G) \leq 3$. Furthermore such a coloring can be produced with the strong property.

The example in Figure 1 may suggest, as was conjectured in [7], that 2-connectivity along with a minimum degree assumption may be enough to force the proper connection number down to 2 but this is not the case.

Theorem 3.3 ([29]) There exists a class of graphs with minimum degree 3 and proper connection number 3 .

This was recently improved further.

Theorem 3.4 ([8]) For every integer $d \geq 3$, there exists a 2-connected graph $G$ of minimum degree $d$ and order $n=42 d$ such that $p c(G)=3$.

In the case of bipartite graphs, it is easier to force the proper connection number down to 2 .

Theorem 3.5 ([7]) If $G$ is a bipartite graph with $\kappa(G) \geq 2$, then $p c(G)=2$. Furthermore, such a coloring can be produced with the strong property.

This result was improved slightly in [30].

Theorem 3.6 ([30]) If $G$ is a connected bipartite bridgeless graph, then $p c(G)=2$. Furthermore such a coloring can be produced with the strong property.

As opposed to just connectivity, there have also been results using diameter to provide density in the graph.

Theorem 3.7 ([7]) If $\operatorname{diam}(G)=2$ and $G$ is 2-connected, then $p c(G)=2$.

We also posed the following conjecture in a previous version of this survey.

Conjecture 3.1 If $\operatorname{diam}(G)=3$ and $G$ is 2-connected, then $p c(G)=2$.

This conjecture was proven in [29].

Theorem 3.8 ([29]) If $G$ is a 2-connected noncomplete graph with diam $(G)=3$, then $p c(G)=2$.

In the case where there are two connected spanning subgraphs that share very few edges, the following bound is known.

Theorem 3.9 ([25]) If $G$ is a graph containing two spanning subgraphs $G_{1}$ and $G_{2}$ such that $\left|E\left(G_{1}\right) \cap E\left(G_{2}\right)\right|=t$, then $p c(G) \leq t+4$. 


\section{Complements}

Looking at the complement of the graph $G$, Huang et al. showed the following results.

Theorem 4.1 ([32]) If $G$ is a graph with $\operatorname{diam}(G) \geq 4$, then $p c(\bar{G})=2$.

Theorem 4.2 ([32]) For a connected noncomplete graph $G$, if $\bar{G}$ does not belong to the following two cases: (i) $\operatorname{diam}(\bar{G})=2,3$, (ii) $\bar{G}$ contains exactly two connected components and one of them is trivial, then $p c(G)=2$.

For the next result, let $G$ be a graph containing a vertex $x$ and define $\operatorname{ecc}(x)$ to be the eccentricity of $x$, that is, the maximum distance from another vertex to $x$. Also let $N_{i}(x)$ denote the set of vertices at distance $i$ from $x$ in $G$.

Theorem 4.3 ([32]) Let $G$ be a connected graph with diam $(G)=3$ and let $x$ be a vertex of $G$ such that $\operatorname{ecc}(x)=3$. We have $p c(\bar{G})=2$ for the two cases (i) $G=P_{4}$ or (ii) $N_{2}(x)=1$ and $N_{3}(x) \geq 2$. For the remaining cases, $p c(\bar{G})$ can be arbitrarily large. Furthermore, if $G$ is triangle-free, then $p c(\bar{G})=2$.

The previous result leads to the following ones.

Corollary 4.4 ([32]) Let $G$ be a triangle-free graph with diam $(G)=2$. If $\bar{G}$ is connected, then $p c(\bar{G})=2$.

Theorem 4.5 ([32]) If $G$ is connected and not complete and $\bar{G}$ is triangle-free, then we have $p c(G)=2$.

Based on these and other results, a Nordhaus-Gaddum type result for the proper connection number was obtained.

Theorem 4.6 ([32]) If both $G$ and $\bar{G}$ are connected, then for all $n \geq 5, p c(G)+p c(\bar{G}) \leq n$ and equality holds if and only if $G$ or $\bar{G}$ is the unique tree with a vertex of degree $n-2$.

\section{$5 \quad$ Degree Conditions}

In this section, we consider results which use assumptions on the degrees or number of edges to force density, thereby driving the proper connection number down.

\section{$5.1 \quad$ Minimum Degree}

When density is provided by a minimum degree assumption, the following result is sharp in terms of the minimum degree assumption.

Theorem 5.1 ([7]) If $G$ is a connected (non-complete) graph with $n \geq 68$ vertices and $\delta(G) \geq n / 4$, then $\operatorname{pc}(G)=2$. 
Although the bound on the minimum degree in Theorem 5.1 is best possible, there is nothing to suggest that the bound $n \geq 68$ is required. In the following conjecture, posed in a previous version of this survey, the bound on $n$ would be the best possible since $p c\left(K_{1,3}\right)=3$.

Conjecture 5.1 If $G$ is a connected (non-complete) graph with $n \geq 5$ vertices and $\delta(G) \geq$ $n / 4$, then $\operatorname{pc}(G)=2$.

This conjecture is almost true, as seen in the following result which solves the conjecture completely.

Theorem 5.2 ([28]) Let $G$ be a connected (noncomplete) graph of order $n \geq 5$. If $G \notin$ $\left\{G_{1}, G_{2}\right\}$ (see Figure 2) and $\delta(G) \geq n / 4$, then $p c(G)=2$.

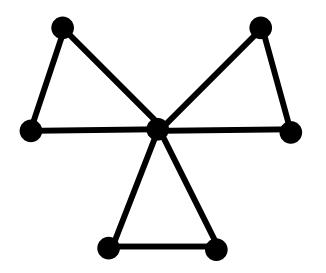

$G_{1}$

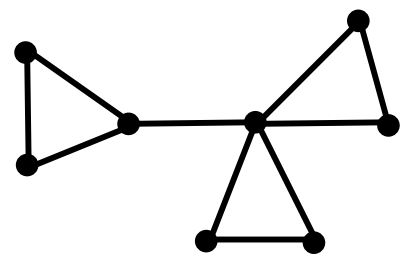

$G_{2}$

Figure 2: Two counterexamples of Conjecture 5.1

The authors of [28] also obtained a similar result for bipartite graphs.

Theorem 5.3 ([28]) Let $G$ be a connected bipartite graph of order $n \geq 4$. If $\delta(G) \geq \frac{n+6}{8}$, then $p c(G)=2$.

Using a weaker minimum degree assumption, the following more general result was obtained.

Theorem 5.4 ([24]) For $t \geq 5$, if $G$ is a connected graph of order $n \geq t^{2}$ and $\delta(G) \geq n / t$, then $p c(G) \leq t-2$.

For bipartite graphs, the natural variation of Theorem 5.4 holds.

Theorem 5.5 ([24]) For $t \geq 4$, if $G$ is a connected bipartite graph of order $n \geq 2 t^{2}$ and $\delta(G)>n / 2 t$, then $p c(G) \leq t-2$.

In general, there are connected graphs with large minimum degree and large proper connection number.

Theorem 5.6 ([8]) For all integers $d, k \geq 2$, there exists a connected graph $G$ with minimum degree $d$ and order $n=(d+1)(k+1)$ such that $p c(G)=k$.

When the graph is 2-connected, it turns out that Theorem 5.1 can be greatly improved.

Theorem 5.7 ([8]) Let $G$ be a 2-connected graph of order $n=n(G)$ and minimum degree $\delta(G) \geq \max \left\{2, \frac{n+8}{20}\right\}$. Then $p c(G) \leq 2$. 


\subsection{Degree Sum}

Let $\mathscr{G}$ be the set of three graphs consisting of the two graphs in Figure 2 along with the graph in which two triangles and an edge all share a single vertex.

Theorem 5.8 ([9]) Let $G$ be a connected noncomplete graph of order $n \geq 5$ with $G \notin \mathscr{G}$. If $d(x)+d(y) \geq \frac{n}{2}$ for every pair of nonadjacent vertices $x, y \in V(G)$, then $p c(G)=2$.

Theorem 5.9 ([9]) Let $G$ be a connected bipartite graph of order $n \geq 4$. If $d(x)+d(y) \geq \frac{n+6}{4}$ for every pair of nonadjacent vertices $x, y \in V(G)$, then $p c(G)=2$.

\subsection{Edge Density}

Using the number of edges in the graph to provide density, the following results hold.

Theorem 5.10 ([30]) If $G$ is a connected graph of order $n \geq 14$ with $\left(\begin{array}{c}n-3 \\ 2\end{array}\right)+4 \leq|E(G)| \leq$ $\left(\begin{array}{l}n \\ 2\end{array}\right)-1$, then $\operatorname{pc}(G)=2$.

Theorem 5.11 ([30]) If $G$ is a connected graph of order $n \geq 15$ with $|E(G)| \geq\left(\begin{array}{c}n-4 \\ 2\end{array}\right)+5$, then $p c(G) \leq 3$.

Let $f(n, k)$ denoted the minimum number of edges such that every connected graph $G$ of order $n$ with at least $f(k, n)$ edges has $p c(G) \leq k$.

Theorem 5.12 ([31]) For any $k \geq 2, f(n, k)=\left(\begin{array}{c}n-k-1 \\ 2\end{array}\right)+k+2$.

With the assumption that $\kappa(G) \geq 2$, the proper connection number can be at most 3 , so in order to produce results using more colors, we must assume that the graph is only 1-connected.

Theorem 5.13 ([24]) For $s \geq 2$, any connected $K_{1, s}$-free graph $G$ has $p c(G) \leq s-1$.

\section{Domination}

Relating the proper connection number with domination, the following results were proven in [40]. Here a subset $D$ of the vertices is called two-way two-step dominating if every pendant vertex is included in $D$ and every vertex at distance at least 2 from $D$ has at least two neighbors at distance 1 from $D$.

Theorem 6.1 ([40]) If $D$ is a connected two-way two-step dominating set of a graph $G$, then $p c(G) \leq p c(G[D])+2$.

This yields the following corollary.

Corollary 6.2 ([40]) If $G$ is a connected graph of order $n \geq 4$ and with minimum degree $\delta$, then $p c(G) \leq \frac{3 n}{\delta+1}-1$. 
A result similar to Theorem 6.1 was shown for two-way dominating sets, namely, a dominating set containing all pendant vertices.

Theorem 6.3 ([40]) If $D$ is a connected two-way dominating set of a graph $G$, then we have $p c(G) \leq p c(G[D])+2$.

Related to these results, the following was proven for a couple of special classes of graphs.

Theorem 6.4 ([40]) If $G$ is a connected interval or circular arc graph with $\delta(G) \geq 2$, then $p c(G) \leq 3$ and this bound is sharp.

\section{Combinations of Graphs}

By a graph join, denoted by $G+H$, we mean adding all possible edges between a copy of $G$ and a copy of $H$. The following was shown for joins.

Theorem 7.1 ([1]) If $G$ and $H$ are nontrivial connected graphs such that $G+H$ is not complete, then $p c(G+H)=2$.

Concerning graph products, we define the Cartesian product of two graphs $G$ and $H$, denoted by $G \square H$, to be the graph with vertex set $V(G) \times V(H)$ and an edge from $(u, v)$ to $\left(u^{\prime}, v^{\prime}\right)$ if and only if either $u=u^{\prime}$ and $v v^{\prime} \in E(H)$ or $v=v^{\prime}$ and $u u^{\prime} \in E(G)$.

Theorem 7.2 ([1]) If $G$ and $H$ are nontrivial connected graphs, then $p c(G \square H)=2$.

In [44], it seems that the authors got a more general result for the relationship between the graphs $G$ and $H$ and their Cartesian product: Let $G$ and $H$ be connected graphs each of order at least 2. Then

$$
p c(G \square H) \leq \min \{p c(G), p c(H)\}+1,
$$

and this bound is sharp. But in fact, they were not aware that actually this result is covered by Theorem 7.2, since for any nontrivial connected graphs $G$ and $H, p c(G) \geq 1$ and $p c(H) \geq 1$.

Other similar results for the strong product, lexicographical product, the direct product as well as applications to grids, meshes, hypercubes and other classes of highly symmetric graphs were proven in [44]. Since these graph products contain the Cartesian product as a spanning subgraph, these results are also covered by Theorem 7.2.

\section{Operations on Graphs}

Given a graph $G$ of order $n$ with vertices $\left\{v_{1}, v_{2}, \ldots, v_{n}\right\}$, let $\alpha$ be a permutation of the set $[n]$. The permutation graph $P_{\alpha}(G)$ is the graph of order $2 n$ obtained from two copies of $G$ by joining a vertex $v_{i}$ in the first copy to the corresponding vertex $u_{i}$ and to $u_{\alpha(i)}$. Note that if $\alpha$ is the identity permutation, then $P_{\alpha}(G)=G \square K_{2}$. The following results were shown for permutation graphs. 
Theorem 8.1 ([1]) If $G$ is a nontrivial graph of order $n$ containing a Hamiltonian path, then $\operatorname{pc}\left(P_{\alpha}\right)=2$ for any permutation $\alpha$ of $[n]$.

Theorem 8.2 ([1]) Every permutation graph of a star of order at least 4 has proper connection number 2.

Given two graphs $G$ and $H$, the direct product, denoted $G \times H$, is the graph with vertex set $V(G) \times V(H)$ and edges of the form $(g, h)\left(g^{\prime}, h^{\prime}\right)$ where $g g^{\prime} \in E(G)$ and $h h^{\prime} \in E(H)$.

Theorem 8.3 ([27]) If $G$ is a connected non-bipartite graph and $H$ is a 2-connected nonbipartite graph, then $p c(G \times H)=2$.

The line graph of a graph $G$, denoted $L(G)$, is the graph whose vertices can be placed in one-to-one correspondence with the edges of $G$ in such a way that two vertices of $L(G)$ are adjacent if and only if the corresponding edges in $G$ are adjacent.

Theorem 8.4 ([2]) For each connected graph $G$ of order at least $3, p c(L(G)) \leq 2$.

For a connected graph $G$ and a positive integer $k$, the $k^{\text {th }}$ power of $G$, denoted $G^{k}$, is the graph with vertex set $V(G)$ and an edge $u v$ if the distinct vertices $u$ and $v$ are at distance at most $k$ in $G$.

Theorem 8.5 ([2]) For each connected graph $G$ of order at least $3, p c\left(G^{2}\right) \leq 2$.

For a given graph $G$, the corona of $G$, denoted $\operatorname{cor}(G)$, is the graph obtained from $G$ by adding a pendant vertex to each vertex of $G$. For $k \geq 2$, the iterated corona graph $\operatorname{cor}^{k}(G)$ is then defined as $\operatorname{cor}\left(\operatorname{cor}^{k-1}(G)\right)$.

Theorem 8.6 ([2]) If $G$ is a nontrivial connected graph and $k$ is a positive integer, then $\max \{p c(G), k\} \leq p c\left(\operatorname{cor}^{k}(G)\right) \leq p c(G)+k$. Furthermore both bounds are best possible.

Theorem 8.7 ([2]) For integers $k \geq 1$ and $n \geq 3$,

$$
p c\left(\operatorname{cor}^{k}\left(K_{n}\right)\right)= \begin{cases}k+1 & \text { if either } k=1 \text { or } k=2 \text { and } n=3, \text { or } \\ k & \text { if either } k=2 \text { and } n \geq 4 \text { or } k \geq 3 .\end{cases}
$$

Theorem 8.8 ([36]) For integers $k \geq 1$ and $n \geq 4$,

$$
p c\left(\operatorname{cor}^{k}\left(C_{n}\right)\right)=\Delta\left(\operatorname{cor}^{k}\left(C_{n}\right)\right)=p c\left(C_{n}\right)+k=k+2 .
$$




\section{Random Graphs}

For random graphs, the following results were shown in [26]. Here let $G(n, p)$ denote the Erdős-Renyi [23] random graph with $n$ vertices and edges appearing with probability $p$. The first result follows easily from the fact that 3 -connected.

Theorem 9.1 ([26]) Almost all graphs have proper connection number 2.

For the next result, it should be noted that if $\alpha(n)$ is sufficiently large, like $c \log \log n$, the result is almost immediate from the fact that the resulting graph will be Hamiltonian with high probability. Thus, the bulk of the work is done in the case where $\alpha(n)$ is tending to infinity very slowly.

Theorem 9.2 ([26]) For sufficiently large $n$, if $p \geq \frac{\log n+\alpha(n)}{n}$ where $\alpha(n) \rightarrow \infty$, then $p c(G(n, p)) \leq 2$.

\section{Proper Distance}

Given a properly connected graph $G$, the proper distance between vertices $u$ and $v$, denoted $\operatorname{pdist}(u, v)$, is the length of the shortest properly colored path from $u$ to $v$. The proper diameter of the graph $G$, denoted $\operatorname{pdiam}(G)$, is the maximum proper distance between two vertices in $G$. Define a fan $F_{1, n}$ to be the graph obtained from the path $P_{n}$ by adding a dominating vertex.

Theorem 10.1 ([18]) For a properly connected 2-coloring of the fan $G=F_{1, n}$ with $n \geq 7$, the proper diameter of $G$ is within $[3, n-1]$ and every integer value in this interval is attainable for some properly connected 2-coloring of $G$.

Theorem 10.2 ([18]) Let $m, n \geq 2$. For a properly connected 2-coloring of a complete bipartite graph $G=K_{m, n}, \operatorname{pdiam}(G) \in\{2,4\}$. Furthermore, there exists a properly connected 2 -coloring with $\operatorname{pdiam}(G)=2$ if and only if $m \leq 2^{n}$.

Proper diameter of some other classes of graphs were considered in [18].

\section{Strong Proper Connection}

The strong proper connection number of a graph $G$, denoted by $\operatorname{spc}(G)$ and defined in [1], is the minimum number of colors needed to color the edges of $G$ so that between every pair of vertices, there is a properly colored geodesic. 


\subsection{General Results}

First the following observations were made.

Proposition 11.1 ([1]) Let $G$ be a nontrivial connected graph of order $n$ and size $m$. Then

1. $\operatorname{spc}(G)=p c(G)=1$ if and only if $G=K_{n}$;

2. $\operatorname{spc}(G)=p c(G)=m$ if and only if $G=K_{1, m}$;

3. if $G$ is a tree, then $\operatorname{spc}(G)=p c(G)=\Delta(G)$;

4. if $G$ is a connected graph with $\operatorname{diam}(G)=2$, then $\operatorname{spc}(G)=\operatorname{src}(G)$;

5. if $b$ is the maximum number of bridges incident to a single vertex in $G$, then $\operatorname{spc}(G) \geq b$.

Clearly $p c(G) \leq \operatorname{spc}(G)$ for any graph $G$. It turns out that the two values can also be arbitrarily far apart.

Theorem 11.1 ([1]) For every pair of integers $a$ and $b$ with $2 \leq a \leq b$, there exists $a$ connected graph $G$ such that $p c(G)=a$ and $\operatorname{spc}(G)=b$.

A similar result holds for the strong proper connection number in relation to the chromatic index.

Theorem 11.2 ([1]) For every triple of integers $a, b$ and $n$ with $2 \leq a \leq b<n$, there exists a connected graph of order $n$ with $\operatorname{spc}(G)=a$ and $\chi^{\prime}(G)=b$.

When the graph has large girth $g(G)$, it turns out that the bound above is sharp.

Proposition 11.2 ([1]) If $G$ is a connected graph with $g(G) \geq 5$, then $\operatorname{spc}(G)=\chi^{\prime}(G)$.

Comparing $\operatorname{spc}(G)$ to $\operatorname{src}(G)$, we have the following results.

Theorem 11.3 ([1]) For every pair of integers $a$ and $b$ with $2 \leq a \leq b$, there exists $a$ connected graph $G$ such that $\operatorname{spc}(G)=a$ and $\operatorname{src}(G)=b$.

The authors of [35] also classified the graphs with strong proper connection number large, that is, close to the number of edges.

Theorem 11.4 ([35]) Let $G$ be a connected graph of size $m \geq 3$. Then $\operatorname{spc}(G)=m-1$ if and only if $G=S_{2, m-1}$.

Theorem 11.5 ([35]) Let $G$ be a connected graph of size $m \geq 4$. Then $\operatorname{spc}(G)=m-2$ if and only if $G$ is the tree with $\Delta(G)=m-2$ or $G \in\left\{C_{4}, C_{5}, K_{1, m-1}+e\right\}$.

Theorem 11.6 ([35]) Let $G$ be a connected graph of size $m \geq 5$. Then $\operatorname{spc}(G)=m-3$ if and only if $G$ is one of the following graphs:

- a tree with $\Delta(G)=m-3$, 
- one of three classes of graphs looking like a star connected to a small cycle, or

- one of five graphs on at most 6 edges.

Regarding unicyclic graphs, the following has been proven.

Theorem 11.7 ([36]) If $G$ is a unicyclic graph of order at least 3, then $\Delta(G)-1 \leq$ $\operatorname{spc}(G) \leq \Delta(G)+1$. Furthermore, if $C$ is the unique cycle of $G$, then

1. $\operatorname{spc}(G)=\Delta(G)+1$ if and only if $G=C$ is an odd cycle of order at least 5 ,

2. $\operatorname{spc}(G)=\Delta(G)$ if and only if

- $G=C$ is an even cycle,

- $\Delta(G) \geq 3$ and $C$ is a cycle of order at least 4 , or

- $C=C_{3}$ and $\operatorname{deg}(u)=\Delta(G)$ for some $u \in V(G \backslash C)$.

3. $\operatorname{spc}(G)=\Delta(G)-1$ if and only if $C=C_{3}$ and $\operatorname{deg}(u)<\Delta(G)$ for all $u \in V(G \backslash C)$.

\subsection{Operations on Graphs}

The following was shown for line graphs of trees.

Theorem 11.8 ([36]) If $T$ is a tree of order at least 3 , then $\operatorname{spc}(L(T)) \leq 2$.

Regarding powers of trees, the following has been proven.

Theorem 11.9 ([36]) If $T$ is a tree with maximum degree $\Delta$, then $\operatorname{spc}\left(T^{2}\right) \leq(\Delta-1)^{2}+1$

Furthermore, the authors of [36] believe the following to be true.

Conjecture 11.1 ([36]) For each tree $T$ that is not a star, $2 \leq \operatorname{spc}\left(T^{2}\right) \leq \Delta(T)$.

\section{Proper Vertex Connection}

Notions of vertex proper connection, the vertex-coloring version of the proper connection number, have been defined and studied independently in [16] and [34]. A vertex-colored graph $G$ is called proper vertex $k$-connected if every pair of vertices is connected by $k$ internally disjoint paths, each of which has no two consecutive internal vertices of the same color. Define the proper vertex $k$-connection number of $G$, denoted by $p v c_{k}(G)$, to be the smallest number of (vertex) colors needed to make $G$ proper vertex $k$-connected.

Some basic observations about $p v c_{k}(G)$ include the following.

Fact 12.1 ([34]) For any graph $G$,

$$
0 \leq p v c_{k}(G) \leq \min \left\{\chi(G), r v c_{k}(G)\right\}
$$

where $\operatorname{rvc}(G)$ is the rainbow vertex connection number. 
Proposition 12.1 ([34]) If $G$ is a nontrivial connected graph, then

- $\operatorname{pvc}(G)=0$ if and only if $G$ is a complete graph, and

- $\operatorname{pvc}(G)=1$ if and only if $\operatorname{diam}(G)=2$.

In particular, the following results were shown for cycles and wheels.

Theorem $12.1([34]) \quad \bullet p v c\left(C_{3}\right)=0, p v c\left(C_{4}\right)=p v c\left(C_{5}\right)=1$, and $p v c\left(C_{n}\right)=2$ for $n \geq 6$.

- $p v c_{2}\left(C_{3}\right)=1, p v c_{2}\left(C_{n}\right)=2$ for even $n \geq 4$ and $p v c_{2}\left(C_{n}\right)=3$ for odd $n \geq 5$.

- $\operatorname{pvc}\left(W_{3}\right)=0$ and $\operatorname{pvc}\left(W_{n}\right)=1$ for $n \geq 4$.

- $p v c_{2}\left(W_{3}\right)=1$ and $p v c_{2}\left(W_{n}\right)=p v c\left(C_{n}\right)$ for all $n \geq 4$.

- $p v c_{3}\left(W_{3}\right)=1$ and $p v c_{3}\left(W_{n}\right)=p v c_{2}\left(C_{n}\right)$ for all $n \geq 4$.

The authors of [34] also have a result for $p v c_{k}$ of complete multipartite graphs. More generally, the following results were shown for general graphs.

Theorem 12.2 ([34]) If $G$ is a nontrivial connected graph, then $p^{2} c_{1}(G)=2$ if and only if $\operatorname{diam}(G) \geq 3$.

Problem 12.1 ([34]) For $k \geq 2$, is it true that $p c_{k}(G) \geq p v c_{k}(G)$ for any connected graph $G$ ?

The work of [34] also considers the notion of strong proper vertex connection which requires that there exists a shortest path between any pair of selected vertices that is internally properly colored.

Theorem 12.3 ([34]) If $G$ is a nontrivial connected graph of order $n$, then $0 \leq \operatorname{spvc}(G) \leq$ $n-2$ and equality holds on the right if and only if $G \in\left\{P_{3}, P_{4}\right\}$.

The authors of [34] also classified the 12 graphs satisfying $\operatorname{spvc}(G)=n-3$. In relation to other related parameters, the following existence results are known.

Theorem 12.4 ([34]) For every pair of integers $a$ and $b$ with $2 \leq a \leq b$, there exists $a$ connected graph $G$ such that $\operatorname{spvc}(G)=a$ and $\operatorname{srvc}(G)=b$.

Theorem 12.5 ([34]) For every triple of integers $a, b$ and $c$ with $2 \leq a \leq b \leq c$, there exists a connected graph $G$ with $\operatorname{spvc}(G)=a, \chi(G)=b$ and $\Delta(G)=c$.

Similarly, the work of [16] considers a slightly different definition in which the entire paths (including end vertices) must be properly colored. Denote this variant $\operatorname{vpc}(G)$. It turns out that this proper connection number can be classified by $S \chi_{k}(G)$, the minimum chromatic number of a spanning $k$-connected subgraph of $G$.

Theorem 12.6 ([16]) Given a k-connected graph $G, v p c_{k}(G)=s \chi_{k}(G)$. 


\section{Total Proper Connection}

Very recently, a notion of total proper connection was introduced in [33]. A graph is total colored if both edges and vertices receive colors. A path in a total colored graph is called a total proper path if no two adjacent edges of the path receive the same color, no two adjacent vertices of the path receive the same color and no vertex of the path receives the same color as an incident edge. A total colored graph $G$ is called total proper connected if every two vertices in $G$ are connected by a total proper path and the total proper connection number of a graph $G$, denoted by $\operatorname{tpc}(G)$, is the minimum number of colors needed to total color $G$ so that the coloring is total proper connected. Preliminary results on the subject include the following.

Proposition 13.1 ([33]) If $G$ is a nontrivial connected graph and $H$ is a connected subgraph of $G$, then $\operatorname{tpc}(G) \leq \operatorname{tpc}(H)$.

Proposition 13.2 ([33]) Let $G$ be a connected graph of order $n \geq 3$ containing at least one bridge. If $b$ is the maximum number of bridges incident with a single vertex, then $\operatorname{tpc}(G) \geq b+1$.

Theorem 13.1 ([33]) If $T$ is a tree of order $n \geq 3$, then $\operatorname{tpc}(T)=\Delta(T)+1$.

These results immediately imply the following.

Corollary 13.2 ([33]) For a nontrivial connected graph $G, \operatorname{tpc}(G) \leq \min \{\Delta(T)+1: T$ is a spanning tree of $G\}$.

More specifically, the following holds when $G$ is traceable.

Corollary 13.3 ([33]) If $G$ is traceable, then $\operatorname{tpc}(G)=3$.

For complete bipartite graphs, the following results were shown to hold.

Theorem 13.4 ([33]) For $2 \leq m \leq n$, we have $\operatorname{tpc}\left(K_{m, n}\right)=3$.

For total colorings, we extend the definition of the strong property (see Definition 3.1) to include the assumption that the first (and similarly last) edges of the paths must additionally not share a color with the starting (respectively ending) vertex. With this, we may state one of the main results of [33].

Theorem 13.5 ([33]) Let $G$ be a 2-connected graph. Then $\operatorname{tpc}(G) \leq 4$ and there exists a total coloring of $G$ with 4 colors with the strong property.

For general (not necessarily 2-connected) graphs the authors of [33] obtained the following result.

Theorem 13.6 ([33]) Let $G$ be a connected graph and $\widetilde{\Delta}(G)$ be the maximum degree of a vertex that is an endpoint of a bridge of $G$. Then $\operatorname{tpc}(G) \leq \widetilde{\Delta}(G)+1$ if $\widetilde{\Delta}(G) \geq 4$ and $\operatorname{tpc}(G) \leq 4$ otherwise. 
Regarding the minimum degree of $G$, the authors of [33] obtained the following result.

Theorem 13.7 ([33]) If $G$ is a connected graph of order $n$ with minimum degree $\delta$, then $\operatorname{tpc}(G) \leq \frac{3 n}{\delta+1}+1$.

Two interesting questions are also included. The first question was answered in the affirmative for the case where $k=3$ in [33], which motivated the question itself.

Question 13.1 ([33]) For $k \geq 4$, does there exist a graph $G$ such that $\operatorname{tpc}(G)=p c(G)=k$ ?

An affirmative solution to the second question for the case when $t \leq 2$ was also noted in [33], motivating the question.

Question 13.2 ([33]) For $t \geq 3$, does there exist a graph $G$ such that $t p c(G)=p c(G)+t$ ?

As a Nordhaus-Gaddum-type result for total proper connection, the following sharp bounds were obtained in [39].

Theorem 13.8 ([39]) If $G$ and $\bar{G}$ are connected complementary graphs on $n$ vertices, then $6 \leq \operatorname{tpc}(G)+\operatorname{tpc}(\bar{G}) \leq n+2$.

The authors of [39] also classified when the number can achieve the upper bound along with some other particular values.

\section{More Paths}

Using a minimum degree assumption to provide density, the following was shown for $p c_{2}(G)$.

Theorem 14.1 ([30]) If $G$ is a connected graph with $n \geq 4$ vertices and $\delta(G) \geq n / 2$, then $p c_{2}(G)=2$.

Not too surprisingly, the natural extension from the minimum degree to the degree sum also holds.

Theorem 14.2 ([30]) If $G$ is a connected graph with $n \geq 4$ vertices and $\sigma_{2}(G) \geq n$, then $p c_{2}(G)=2$.

With high connectivity in the underlying (uncolored) graph, it appears as though the $k$-proper connection number stays small.

Conjecture 14.1 ([7]) If $k \geq 2$ and $G$ is $2 k$-connected, then $p c_{k}(G) \leq 3$.

The case of Conjecture 14.1 where $k=1$ follows from Theorem 3.1.

Proposition 14.1 ([7]) If $k \geq 1, p=2 k-1$ and $q>2^{p}$, then $p c_{k}\left(K_{p, q}\right)>2$.

In light of this proposition, the following conjecture was made. 
Conjecture 14.2 ([7]) If $G$ is a $2 k$-connected bipartite graph with $k>1$, then $p c_{k}(G)=2$.

This conjecture holds for complete bipartite graphs, as seen in the following result, but the general case remains open.

Theorem 14.3 ([7]) If $G=K_{n, m}, m \geq n \geq 2 k$ for $k \geq 1$, then $p c_{k}(G)=2$.

For the specific complete bipartite graphs $K_{3, n}$, the proper connection number for two paths is completely solved.

Theorem 14.4 ([7]) If $G=K_{3, n}$, then

$$
p c_{2}(G)= \begin{cases}2 & \text { if } 3 \leq n \leq 6 \\ 3 & \text { if } 7 \leq n \leq 8 \\ \lceil\sqrt[3]{n} & \text { if } n \geq 9\end{cases}
$$

For complete graphs, the following is known for any number of paths.

Theorem 14.5 ([7]) If $G=K_{n}$ with $n \geq 4, k>1$ and $n \geq 2 k$, then $p c_{k}(G)=2$.

For complete bipartite graphs, the following have been shown.

Theorem 14.6 ([37]) If $r, s$ and $k$ are integers with $2 \leq r \leq s$ and $2 \leq k \leq r \leq 2 k$, then $p c_{k}\left(K_{s, t}\right) \geq\lceil\sqrt[2 r-2 k+1]{s}\rceil$.

Theorem 14.7 ([37]) If $k, r$ and $s$ are integers with $k \geq 2, r=2 k-1$ and $s \geq 3^{r}$, then $p c_{k}\left(K_{r, s}\right)=\lceil\sqrt[r]{s}\rceil$

Theorem 14.8 ([37]) If $k, r$ and $s$ are integers with $k \geq 3, r=2 k-2$ and $s \geq 3^{r-1}$, then $p c_{k}\left(K_{r, s}\right)=\lceil\sqrt[r-1]{s}\rceil$

\section{Directed Graphs}

Much like the undirected version, a strongly connected directed graph is called properly connected if between every ordered pair of vertices, there is a directed properly colored path. Defined in [43], the directed proper connection number of a strongly connected directed graph $G$, denoted by $\overrightarrow{p c}(G)$, is the minimum number of colors needed to color the (directed) edges so that the graph is properly connected. Clearly $\overrightarrow{p c}(G) \geq 2$ for any $G$ since a directed edge from $u$ to $v$ implies there is no directed edge from $v$ to $u$ so a directed path from $v$ to $u$ must use at least 2 colors.

It turns out that this number is always at most 3 .

Theorem 15.1 ([43]) If $G$ is strongly connected, then $\overrightarrow{p c}(G) \leq 3$.

In the case of a tournament, the number is always 2. 
Theorem 15.2 ([43]) If $G$ is a strongly connected tournament, then $\overrightarrow{p c}(G)=2$.

The authors of [43] left the following problem open.

Problem 15.1 ([43]) Classify the graphs with $\overrightarrow{p c}(G)=2$.

They also asked whether each of the graphs in the above classification contain a strongly connected bipartite subgraph which spans all but at most two vertices. As a special case of such a result, there is also the following conjecture.

Conjecture 15.1 ([43]) If $G$ is a strongly connected digraph with no even directed cycle, then $\overrightarrow{p c}(G)=3$.

With regards to color degree, the following was shown.

Theorem 15.3 ([43]) If in a colored tournament $G$ of odd order $n \geq 201$, each vertex has $\frac{n-1}{2}$ different colors on in-edges and $\frac{n-1}{2}$ different colors on out-edges, then $G$ is properly connected.

The bound on the number of colors on the in or out edges is actually best possible while the bound on $n$ is likely not necessary.

Conjecture 15.2 (Implied in [43]) If in an edge-colored tournament $G$ of odd order $n$, each vertex has $\frac{n-1}{2}$ different colors on in-edges and $\frac{n-1}{2}$ different colors on out-edges, then $G$ is properly connected.

\section{Generalizations}

There have been some generalizations or extensions of the proper connection number.

\section{$16.1(k, \ell)$-Rainbow Connectivity}

A path is called $\ell$-rainbow if every subpath of length at most $\ell+1$ is rainbow. Defined in [41] under a different title and [12] with a slightly different definition, the $(k, \ell)$-rainbow connection number, denoted $r c_{k, \ell}(G)$, is the minimum number of colors needed to color the edges of $G$ so that between every pair of vertices, there are at least $k$ internally disjoint $\ell$-rainbow paths.

First some easy observations.

Proposition 16.1 ([41]) Let $G$ be a nontrivial connected graph and $\ell \geq 1$ be an integer. Then we have $p c(G) \leq r c_{1,2} \leq \cdots \leq r c_{1, \ell-1} \leq r c_{1, \ell}(G) \leq r c(G)$.

Proposition 16.2 ([41]) If $G$ is a nontrivial connected graph, $H$ is a connected spanning subgraph of $G$ and $\ell \geq 1$ is an integer, then $r c_{1, \ell}(G) \leq r c_{1, \ell}(H)$.

Proposition 16.3 ([41]) Let $G$ be a traceable graph and $\ell$ be a positive integer then we have $r c_{1, \ell}(G) \leq \ell+1$. 
It turns out that the $(1,2)$-rainbow connection number of a tree is almost the largest sum of degrees of adjacent vertices, denoted $\sigma_{2}^{\prime}(G)$.

Theorem 16.1 ([41]) If $T$ is a nontrivial tree, then $r c_{1,2}(T)=\sigma_{2}^{\prime}(T)-1$.

For complete bipartite graphs, the following is known.

Theorem $16.2([41])$ Let $\ell \geq 2$ be an integer and $m \leq n$. Then,

$$
r c_{1, \ell}\left(K_{m, n}\right)= \begin{cases}n & \text { if } m=1, \\ 2 & \text { if } m \geq 2 \text { and } m \leq n \leq 2^{m}, \\ 3 & \text { if } \ell=2, m \geq 2 \text { and } n>2^{m}, \text { or } \\ & \ell \geq 3, m \geq 2 \text { and } 2^{m}<n \leq 3^{m} \\ 4 & \text { if } \ell \geq 3, m \geq 2 \text { and } n>3^{m}\end{cases}
$$

Then for complete multipartite graphs, the following is known.

Theorem $16.3([41])$ Let $t \geq 3, \ell \geq 1,1 \leq n_{1} \leq \cdots \leq n_{t}, m=\sum_{i=1}^{t-1} n_{i}$ and $n=n_{t}$. Then,

$$
r c_{1, \ell}\left(K_{n_{1}, \cdots, n_{t}}\right)= \begin{cases}1 & \text { if } n=1 \\ 2 & \text { if } 2 \leq n \leq 2^{m} \\ 3 & \text { otherwise. }\end{cases}
$$

For wheels, we have the following.

Theorem 16.4 ([41]) Let $n \geq 3$ and $\ell \geq 2$, then,

$$
\operatorname{rc}_{1, \ell}\left(W_{n}\right)= \begin{cases}1 & \text { if } n=3 \\ 2 & \text { if } 4 \leq n \leq 6 \\ 3 & \text { otherwise }\end{cases}
$$

If we let $Q_{t}$ denote the $t$-dimensional hypercube, the following holds.

Theorem 16.5 ([41]) Let $t \geq 1$ and $\ell \geq 2$. Then

$$
r c_{1, \ell}\left(Q_{t}\right)=\left\{\begin{array}{cl}
1 & \text { if } t=1 \\
2 & \text { if } t=2, \\
t & \text { if } t \geq 3 \text { and } \ell \geq t \\
\ell+1 & \text { if } t \geq 3 \text { and } \ell<t .
\end{array}\right.
$$

When the graph is 2-connected, the $(1,2)$-rainbow connection number is at most 5 .

Theorem 16.6 ([41]) If a graph $G$ is 2-connected, then $\operatorname{rc}_{1,2}(G) \leq 5$.

The authors of [41] also considered some graph operations, the join, the Cartesian product, and permutation graphs. 
Theorem 16.7 ([41]) If $G$ and $H$ are nontrivial connected graphs, then $\operatorname{rc}_{1,2}(G+H) \leq 3$ where ' + ' denotes the standard graph join, adding all edges between $G$ and $H$.

Theorem 16.8 ([41]) Let $G$ and $H$ be nontrivial connected graphs, and not both of them are complete graphs. Then

(i) $r c_{1,2}(G \square H)=3$ except if $G($ or $H)$ is a star and every spanning tree of $H$ (respectively $G$ ) has radius at least 3.

(ii) $r c_{1,2}(G \square H) \leq 4$ if $G($ or $H)$ is a star and every spanning tree of $H$ (respectively $G$ ) has radius at least 3 .

Theorem 16.9 ([41]) Let $G$ be a nontrivial traceable graph of order $n$, then

$$
r c_{1, \ell}\left(P_{\alpha}(G)\right) \leq \ell+1
$$

for each permutation $\alpha$ of $[n]$.

Concerning graphs and their complements, the following results hold.

Theorem 16.10 ([42]) If $G$ is a connected graph with diam $(\bar{G}) \geq 4$, then $\operatorname{rc}_{1,2}(G) \leq 3$.

Theorem 16.11 ([42]) If $G$ is a connected graph such that $\bar{G}$ is triangle-free and additionally $\operatorname{diam}(\bar{G})=3$, then $\operatorname{rc}_{1,2}(G)=3$.

Theorem 16.12 ([42]) If $G$ is a connected graph such that $\bar{G}$ is triangle-free and additionally $\operatorname{diam}(\bar{G})=2$, then $\operatorname{rc}_{1,2}(G) \leq 3$.

The authors of [42] also classified those graphs $G$ with $r c_{1,2}(G)=n-1$ or with $r c_{1,2}(G)=$ $n-2$. This result led to the following Nordhaus-Gaddum type result.

Theorem 16.13 ([42]) Let $G$ and $\bar{G}$ be connected graphs on $n$ vertices. Then $r c_{1,2}(G)+$ $r c_{1,2}(\bar{G}) \leq n+2$ and the equality holds if and only if $G$ or $\bar{G}$ is isomorphic to a double star.

For complete bipartite graphs, the following has been shown.

Theorem $16.14([12,19])$ Let $s$ and $t$ be integers with $2 \leq s \leq t$ and let $\ell$ be the length of a longest path in $K_{s, t}$. If $k$ is and integer with $4 \leq k \leq \ell$, then $r c_{1, k+1}\left(K_{s, t}\right)=\min \{\lceil\sqrt[s]{t} \mid, 4\}$.

The authors of [19] also explored other special classes of graphs.

\subsection{Proper Hamiltonian-Connection}

An edge-colored graph $G$ is called a proper Hamiltonian-path coloring if between every pair of vertices, there is a properly colored Hamiltonian path. The proper Hamiltonian-connection number of $G$, denoted $h p c(G)$, is then defined to be the minimum number of colors needed to color $G$ to be a proper Hamiltonian-path coloring. Defined in [5], the following results were shown along with some special classes of graphs.

Theorem $16.15([5])$ For all $n \geq 4, h p c\left(K_{n}\right)=2$.

Theorem 16.16 ([5]) For each odd integer $n \geq 3$, $h p c\left(C_{n} \square K_{2}\right)=3$. 


\subsection{Proper-Walk Connection}

As opposed to a properly colored path, we may also consider a properly colored walk. The proper-walk connection number, denoted $p W(G)$, is defined to be the minimum number of colors needed to color the edges of $G$ such that between every pair of vertices, there is a properly colored walk. Several classes of graphs have been considered.

Theorem 16.17 ([45]) Let $G$ be a connected graph that is not a tree. Then $p W(G) \leq 3$.

For a graph $G$, define $M(G)$ as the set of all maximal 2-edge-connected subgraphs of $G$.

Theorem 16.18 ([45]) Let $G$ be a connected bipartite graph. Then $p W(G)=2$ if and only if every subgraph in $M(G)$ is incident with at most two bridges.

Theorem 16.19 ([45]) If a connected graph $G$ has two edge-disjoint odd cycles, then we have $p W(G)=2$.

Theorem 16.20 ([45]) If $G$ is a connected bridgeless graph, then $p W(G) \leq 2$.

Theorem 16.21 ([45]) Let $G$ be a graph formed by adding pendant edges to the vertices of an odd cycle. Then $p W(G)=2$ if and only if there are three consecutive vertices $u, v, w$ on the cycle such that $u$ and $w$ are incident to at most one pendant edge each, and all vertices other than $u, v, w$ are incident to no pendant edges.

The authors of [45] also include a brief discussion of $p W$ for directed graphs.

\subsection{Proper Index}

An edge-colored tree $T$ is called a proper tree if no two adjacent edges share a color. Given an edge-colored graph $G$ and a subset of the vertices $S \subseteq V(G)$, a tree $T \subseteq G$ is called an $S$-tree if it contains the vertices of $S$. An edge-coloring of $G$ is called a $k$-proper coloring if for every $k$-subset $S$, there is a proper $S$-tree as a subgraph of $G$. For a connected graph $G$, the $k$-proper index of $G$, denoted $p x_{k}(G)$, is the minimum number of colors needed to color $G$ to make a $k$-proper coloring.

From the definition, the following are clear.

Fact 16.1 ([15]) For any graph $G$ of order $n \geq 3$,

$$
p c(G)=p x_{2}(G) \leq p x_{3}(G) \leq \cdots \leq p x_{n}(G)
$$

Proposition 16.4 ([15]) If $G$ is a connected graph of order $n \geq 3$ and $H$ is a connected spanning subgraph of $G$, then $p x_{k}(G) \leq p x_{k}(H)$ for any $k$ with $3 \leq k \leq n$.

Proposition 16.5 ([15]) Let $G$ be a connected graph of order $n \geq 3$ with edge-chromatic number $\chi^{\prime}(G)$. Then for every integer $k$ with $3 \leq k \leq n$, we have $p x_{k}(G) \leq \chi^{\prime}(G) \leq$ $\Delta(G)+1$. 
Proposition 16.6 ([15]) Let $G$ be a connected graph of order $n \geq 3$. Then $p x_{k}(G) \geq 2$ for any integer $k$ with $3 \leq k \leq n$. Furthermore there are infinitely many graphs satisfying this lower bound for each $k$.

Related to this result, the authors of [15] posed the following problem.

Problem 16.1 ([15]) For each $k$, classify those graphs $G$ for which $p x_{k}(G)=2$.

In particular, the following is known.

Proposition 16.7 ([15]) Let $G$ be a connected graph of order $n \geq 3$. Then $p x_{n}(G)=2$ if and only if $G$ is a traceable graph.

For some specific graph classes, the following are known.

Proposition 16.8 ([15]) Let $K_{n}, C_{n}$ and $W_{n}$ be the complete graph, the cycle and the wheel respectively of order $n$. Then for any integer $k$ with $3 \leq k \leq n$, we have $p x_{k}\left(K_{n}\right)=$ $p x_{k}\left(C_{n}\right)=p x_{k}\left(W_{n}\right)=2$.

Proposition 16.9 ([15]) Let $G$ be a unicyclic graph of order $n \geq 3$. Then for each integer $k$ with $3 \leq k \leq n, p x_{k}(G)=\Delta(G)-1$.

The authors of [15] also classify those graphs with $k$-proper index equal to $n-1$ or $n-2$.

For complete bipartite graphs, the following results complete the study of the 3-proper index.

Theorem 16.22 ([38]) For any integer $t \geq 2$, we have

$$
p x_{3}\left(K_{2, t}\right)= \begin{cases}2 & \text { if } 2 \leq t \leq 4 \\ 3 & \text { if } 5 \leq t \leq 18 \\ {\left[\sqrt{\frac{t}{2}}\right.} & \text { if } t \geq 19\end{cases}
$$

Theorem 16.23 ([38]) For any integer $t \geq 3$, we have

$$
p x_{3}\left(K_{3, t}\right)= \begin{cases}2 & \text { if } 3 \leq t \leq 12 \\ 3 & \text { if } t \geq 13\end{cases}
$$

Theorem 16.24 ([38]) For a complete bipartite graph $K_{s, t}$ with $t \geq s \geq 4$, we have $p x_{3}\left(K_{s, t}\right)=2$.

For complete multipartite graphs, the following holds. 
Theorem 16.25 ([38]) Let $G=K_{n_{1}, n_{2}, \ldots, n_{r}}$ be a complete multipartite graph where $r \geq 3$ and $n_{1} \leq n_{2} \leq \cdots \leq n_{r}$. Set $s=\sum_{i=1}^{r-1} n_{i}$ and $t=n_{r}$. Then

$$
p x_{3}(G)= \begin{cases}3 & \text { if } G=K_{1,1, t}, 5 \leq t \leq 18, \\ & \text { or } G=K_{1,2, t}, t \geq 13, \\ & \text { or } G=K_{1,1,1, t}, t \geq 15, \\ \left\lceil\sqrt{\frac{t}{2}} \quad\right. & \text { if } G=K_{1,1, t}, t \geq 19 \\ 2 & \text { otherwise. }\end{cases}
$$

For general $k$, the following hold.

Theorem 16.26 ([38]) For $t \geq s \geq k$, we have $2 \leq p x_{k}\left(K_{s, t}\right) \leq 3$. For $t \geq s \geq 2(k-1)$, we have $\operatorname{px}_{k}\left(K_{s, t}\right)=2$.

Theorem 16.27 ([38]) Let $G=K_{n_{1}, n_{2}, \ldots, n_{r}}$ be a complete multipartite graph where $r \geq 3$ and $n_{1} \leq n_{2} \leq \cdots \leq n_{r}$. Set $s=\sum_{i=1}^{r-1} n_{i}$ and $t=n_{r}$. If $t \geq s \geq 2(k-1)$ or $t \leq s$, then we have $\operatorname{px}_{k}(G)=2$.

For $k=3$ but more general graphs, the following result holds.

Theorem 16.28 ([11]) Let $G$ be a 2-connected non-Hamiltonian graph of order $n$ with $n \geq 4$. Then $p x_{3}(G) \leq \frac{n}{2}$.

The authors of [11] also proved sharp bounds on $p x_{3}(G)$ based on 3-way dominating sets. A dominating set $D$ in a graph $G$ is called an 3-way dominating set if every vertex of $\bar{D}$ has at least 3 neighbours in $G$. In addition, if $G[D]$ is connected, we call $D$ a connected 3-way dominating set. A set $D \subseteq G$ is called an 3-dominating set of $G$ if every vertex of $\bar{D}$ is adjacent to at least 3 distinct vertices of $D$. Furthermore, if $G[D]$ is connected, then $D$ is called a connected 3 -dominating set.

Theorem 16.29 ([11]) If $D$ is a connected 3-way dominating set of a connected graph $G$, then $p_{3}(G) \leq p x_{3}(G[D])+3$. Moreover, this bound is sharp.

This yields the following corollary.

Corollary 16.30 ([11]) Let $G$ be a connected graph with minimum degree $\delta(G) \geq 3$. Then, $p x_{3}(G) \leq n \frac{\ln (\delta+1)}{\delta+1}\left(1+o_{\delta}(1)\right)+2$.

A result similar to Theorem 16.29 was shown for 3-dominating sets.

Theorem 16.31 ([11]) If $D$ is a connected 3-dominating set of a connected graph $G$ with minimum degree $\delta(G) \geq 3$, then $p x_{3}(G) \leq p x_{3}(G[D])+1$. Moreover, the bound is sharp.

Related to these results, the following was proven for a couple of special classes of graphs. 
Theorem 16.32 ([11]) Let $G$ be a connected threshold or chain graph with $\delta(G) \geq 3$. Then, $p x_{3}(G) \leq 3$, and the bound is sharp.

Define the $(k, \ell)$-proper index of a graph $G$, denoted $p x_{k, \ell}(G)$, to be the minimum number of colors needed to color the edges of $G$ so that for every set $S \subseteq V(G)$ of $k$ vertices, there are $\ell$ internally disjoint proper $S$-trees. Even for complete graphs, this problem is already tricky. The following have been shown.

Theorem $16.33([\mathbf{1 0}])$ For $n \geq 4$, we have $p x_{3,2}\left(K_{n}\right)=2$ and $p x_{n-1,2}\left(K_{n}\right)=2$.

Theorem 16.34 ([10]) For two integers $n$ and $\ell$ with $n \geq 2 \ell>0, p x_{n, \ell}\left(K_{n}\right)=2$.

Theorem 16.35 ([10]) Let $k \geq 3$ and $\ell \geq 1$. For all $n$ with

$$
n \geq 2 \frac{(\ell(k-1)+k)^{4}-1}{(\ell+1)(k-1)}-1
$$

we have $p x_{k, \ell}\left(K_{n}\right)=2$.

Theorem 16.36 ([10]) Let $k \geq 3$ and $\ell \geq 1$. For all $n$ with

$$
n \geq 2 k(k+\ell) \ln \left(\frac{1}{1-(1 / 2)^{2 k-3}}\right),
$$

we have $p x_{k, \ell}\left(K_{n}\right)=2$.

For complete bipartite graphs, the following has been shown.

Theorem 16.37 ([10]) Let $k$ and $\ell$ be two positive integers with $k \geq 3$. Then there exists a positive integer $N$ such that $p x_{k, \ell}\left(K_{n, n}\right)=2$ for every integer $n \geq N$.

For random graphs, the following has been shown.

Theorem $16.38([10])$ Let $a=1+\frac{1}{2^{2 k-3}-1}$ and $c \geq 5$. For every $p \geq c \sqrt[k]{\frac{\log _{a} n}{n}}, p x_{k, \ell}\left(G_{n, p}\right) \leq$ 2 holds almost surely.

\subsection{Minimum Spanning Subgraphs}

Given a connected graph $G$ with $p c(G)=2$, let $\mu(G)$ denote the minimum number of edges in a connected spanning subgraph $H$ of $G$ with $p c(H)=2$.

Theorem $16.39([6])$ For an integer $t \geq 4, \mu\left(K_{2, t}\right)=2 t-2$.

Theorem 16.40 ([6]) If $s$ and $t$ are integers with $t \geq s+2 \geq 5$, then $\mu\left(K_{s, t}\right)=2 t-2$.

Theorem 16.41 ([6]) Let $G=K_{n_{1}, n_{2}, \ldots, n_{k}}$ be a complete $k$-partite graph, where $k \geq 3$ and $r=\sum_{i=1}^{k-1} n_{i} \geq 3$. If $n_{k} \geq r^{2}+r$, then $\mu(G)=2 n_{k}-2 r+2$. 


\section{References}

[1] E. Andrews, E. Laforge, C. Lumduanhom, and P. Zhang. On proper-path colorings in graphs. J. Combin. Math. Combin. Comput., 97: 189-207, 2016.

[2] E. Andrews, E. Laforge, C. Lumduanhom, and P. Zhang. Proper-path colorings in graph operations. Manuscript.

[3] J. Bang-Jensen and G. Gutin. Alternating cycles and paths in edge-coloured multigraphs: a survey. Discrete Math., 165/166:39-60, 1997. Graphs and combinatorics (Marseille, 1995).

[4] J. Bang-Jensen and G. Gutin. Digraphs. Springer Monographs in Mathematics. Springer-Verlag London, Ltd., London, second edition, 2009. Theory, algorithms and applications.

[5] Z. Bi, A. Byers, and P. Zhang. Proper Hamiltonian-connected graphs. Manuscript.

[6] Z. Bi, G. Chartrand, G. Johns, and P. Zhang. On minimum spanning subgraphs of graphs with proper connection number 2. Manuscript.

[7] V. Borozan, S. Fujita, A. Gerek, C. Magnant, Y. Manoussakis, L. Montero, and Z. Tuza. Proper connection of graphs. Discrete Math., 312(17):2550-2560, 2012.

[8] C. Brause, T. D. Doan, and I. Schiermeyer. Minimum degree conditions for the proper connection number of graphs. Manuscript.

[9] H. Chang, Z. Huang, and X. Li. Degree sum conditions for graphs to have proper connection number 2. arXiv:1611.09500v1.

[10] H. Chang, X. Li, C. Magnant, and Z. Qin. The $(k, \ell)$-proper index of graphs. arXiv:1606.03872v2.

[11] H. Chang, X. Li, and Z. Qin. Some upper bounds for the 3-proper index of graphs. Bull. Malays. Math. Sci. Soc., DOI 10.1007/s40840-016-0404-5, in press.

[12] G. Chartrand, S. Devereaux, and P. Zhang. Color-connection and information-transfer paths. Manuscript.

[13] G. Chartrand, G. L. Johns, K. A. McKeon, and P. Zhang. Rainbow connection in graphs. Math. Bohem., 133(1):85-98, 2008.

[14] G. Chartrand, L. Lesniak, and P. Zhang. Graphs $\&$ digraphs. CRC Press, Boca Raton, FL, fifth edition, 2011.

[15] L. Chen, X. Li, and J. Liu. The k-proper index of graphs. Appl. Math Comput., 296:57-63, 2017.

[16] E. Chizmar, C. Magnant, and P. Salehi Nowbandegani. Note on vertex and total proper connection numbers. Manuscript. 
[17] W. S. Chou, Y. Manoussakis, O. Megalakaki, M. Spyratos, and Zs. Tuza. Paths through fixed vertices in edge-colored graphs. Math. Inform. Sci. Humaines, (127):49-58, 1994.

[18] V. Coll, J. Hook, C. Magnant, K. McCready, and K. Ryan. Proper diameter of graphs. Manuscript.

[19] S. Devereaux, G. Johns, and P. Zhang. Color connection in graphs intermediate to proper and rainbow connection. Manuscript.

[20] D. Dorninger. On permutations of chromosomes. In Contributions to general algebra, 5 (Salzburg, 1986), pages 95-103. Hölder-Pichler-Tempsky, Vienna, 1987.

[21] D. Dorninger. Hamiltonian circuits determining the order of chromosomes. Discrete Appl. Math., 50(2):159-168, 1994.

[22] D. Dorninger and W. Timischl. Geometrical constraints on Bennet's predictions of chromosome order. Heredity, 58:321-325, 1987.

[23] P. Erdős and A. Rényi. On the evolution of random graphs. Magyar Tud. Akad. Mat. Kutató Int. Közl., 5:17-61, 1960.

[24] S. Fujita, A. Gerek, and C. Magnant. Proper connection with many colors. J. Comb., 3(4):683-693, 2012.

[25] R. Gu, X. Li, and Z. Qin. Proper connection number of random graphs. arXiv: 1505.0464604 .

[26] R. Gu, X. Li, and Z. Qin. Proper connection number of random graphs. Theoret. Comput. Sci., 609:336-343, 2016.

[27] R. H. Hammack and D. T. Taylor. Proper connection of direct products. Manuscript.

[28] F. Huang, X. Li, Z. Qin, and C. Magnant. Minimum degree condition for proper connection number 2. Theoret. Comput. Sci., DOI 10.1016/j.tcs.2016.04.042, in press.

[29] F. Huang, X. Li, Z. Qin, C. Magnant, and K. Ozeki. On two conjectures about the proper connection number of graphs. arXiv:1602.07163v3.

[30] F. Huang, X. Li, and S. Wang. Proper connection number and 2-proper connection number of a graph. arXiv:1507.01426.

[31] F. Huang, X. Li, and S. Wang. Upper bounds of proper connection number of graphs. J. Comb. Optim., DOI 10.1007/s10878-016-0056-2, in press.

[32] F. Huang, X. Li, and S. Wang. Proper connection number of complementary graphs. Bull. Malays. Math. Sci. Soc., DOI 10.1007/s40840-016-0381-8, in press.

[33] H. Jiang, X. Li, and Y. Zhang. Total proper connection of graphs. arXiv:1512.00726.

[34] H. Jiang, X. Li, Y. Zhang, and Y. Zhao. On (strong) proper vertex-connection of graphs. Bull. Malays. Math. Sci. Soc., DOI 10.1007/s40840-015-0271-5, in press. 
[35] E. Laforge, C. Lumduanhom, and P. Zhang. Characterizations of graphs having large proper connection numbers. Discuss. Math. Graph Theory, 36(2): 439-454, 2016.

[36] E. Laforge, C. Lumduanhom, and P. Zhang. Chromatic-connection in graphs. Congressus Numerantium, to appear.

[37] E. Laforge and P. Zhang. Bounds for proper $k$-connectivity of complete bipartite graphs. Manuscript.

[38] W. Li, X. Li, and J. Zhang. The $k$-proper index of complete bipartite and complete multipartite graphs. arXiv:1608.00105v3.

[39] W. Li, X. Li, and J. Zhang. Nordhaus-Gaddum-type theorem for total proper connection number of graphs. arXiv:1611.08990v2.

[40] X. Li, M. Wei, and J. Yue. Proper connection number and connected dominating sets. Theoretical Comp. Sci., 607:480-487, 2015.

[41] X. Li, C. Magnant, M. Wei, and X. Zhu. Distance proper connection of graphs. arXiv:1606.0654\%.

[42] X. Li, C. Magnant, M. Wei, and X. Zhu. Generalized rainbow connection of graphs and their complements. Discuss. Math. Graph Theory, to appear.

[43] C. Magnant, P. R. Morley, S. Porter, P. Salehi Nowbandegani, and H. Wang. Directed proper connection of graphs. Manuscript.

[44] Y. Mao, F. Yanling, Z. Wang, and C. Ye. Proper connection number and graph products. arXiv:1505.02246.

[45] R. Melville and W. Goddard. Coloring graphs to produce properly colored walks. Manuscript.

[46] V. G. Vizing. On an estimate of the chromatic class of a p-graph. Diskret. Analiz No., 3:25-30, 1964. 